\title{
Coronary sinus electrogram characteristics predicts termination of AF with ablation and long-term clinical outcome.
}

\author{
Shohreh Honarbakhsh ${ }^{1}$, Richard Schilling ${ }^{2}$, Emily Keating ${ }^{1}$, Malcolm Finlay ${ }^{3}$, and Ross \\ Hunter $^{4}$ \\ ${ }^{1}$ Barts Heart Centre, Barts Health NHS trust \\ ${ }^{2}$ Barts and The London NHS Trust \\ ${ }^{3}$ Barts and the London NHS Trust \\ ${ }^{4}$ Queen Mary University of London Barts and The London School of Medicine and \\ Dentistry
}

November 13, 2021

\begin{abstract}
Introduction- Markers predicting AF termination and freedom from AF/atrial tachycardia (AT) has been proposed. The role of CS electrogram characteristics has not yet been evaluated. Methods- Patients undergoing ablation for persistent AF as part of the Stochastic Trajectory Analysis of Ranked signals mapping study were included. Novel CS electrogram characteristics including CS cycle length variability (CLV) and CS activation pattern stability (APS) and proportion of low voltage zones (LVZs) were reviewed as potential predictors for AF termination on ablation and freedom from AF/AT during follow-up. The relationship between localized driver characteristics and CS electrogram characteristics were also assessed. Results- Sixty-five patients were included. AF termination was achieved in 51 patients and $80 \%$ of patients were free from AF/AT during a followup of $29.5 \pm 3.7$ months. CS CLV of $<30 \mathrm{~ms}$, CS APS of [?]30\% and proportion of LVZ $<30 \%$ showed a high diagnostic accuracy in predicting AF termination on ablation and freedom from AF/AT during follow-up (CS CLV OR 25.6, AUC 0.91; CS APS OR 15.9, AUC 0.94; proportion of LVZs OR 21.4, AUC 0.88). These markers were independent predictors of AF termination on ablation and AF/AT recurrence during follow-up. Ablation of a smaller number of drivers that demonstrate greater dominance strongly correlate with greater CS organization. Conclusions- Novel CS electrogram characteristics were independent predictors of AF termination and AF/AT recurrence during follow-up. These markers can potentially aid in predicting outcomes and guide ablation and follow-up strategies.
\end{abstract}

Coronary sinus electrogram characteristics predicts termination of AF with ablation and long-term clinical outcome.

Shohreh Honarbakhsh* MRCP PhD BSc, Richard J Schilling* MRCP MD, Emily Keating ${ }^{*}$ BAppSc IBHRE CCDS, Malcolm Finlay ${ }^{*}$ MRCP PhD BA(Oxon), Ross J Hunter* FESC PhD

*The Barts Heart Centre, St Bartholomew's Hospital, Barts Health NHS trust, London, United Kingdom.

BRIEF TITLE- CS activation predicts ablation response in AF.

WORD COUNT: 4229

FUNDING- British Heart Foundation project grant (PG/16/10/32016).

DISCLOSURES- Prof. Schilling has received speaker and travel grants from Biosense Webster and research grants from Biosense Webster and Boston Scientific. Prof. Hunter has received speaker fees, educational and 
research grants from Medtronic and Biosense Webster. Dr Honarbakhsh, Prof Schilling, Dr Finlay, and Prof. Hunter are shareholders in Rhythm AI Ltd.

Corresponding Author:

Prof Ross J Hunter FESC PhD

Director of Arrhythmia Research

Barts Heart Centre, Barts Health NHS trust

West Smithfield, EC1A 7BE

Fax: +442075738838

Email: ross.hunter3@nhs.net

\section{ABSTRACT}

Introduction- Markers predicting AF termination and freedom from AF/atrial tachycardia (AT) has been proposed. The role of CS electrogram characteristics has not yet been evaluated.

Methods- Patients undergoing ablation for persistent AF as part of the Stochastic Trajectory Analysis of Ranked signals mapping study were included. Novel CS electrogram characteristics including CS cycle length variability (CLV) and CS activation pattern stability (APS) and proportion of low voltage zones (LVZs) were reviewed as potential predictors for AF termination on ablation and freedom from AF/AT during followup. The relationship between localized driver characteristics and CS electrogram characteristics were also assessed.

Results- Sixty-five patients were included. AF termination was achieved in 51 patients and $80 \%$ of patients were free from AF/AT during a follow-up of $29.5 \pm 3.7$ months. CS CLV of $<30 \mathrm{~ms}$, CS APS of [?] $30 \%$ and proportion of LVZ $<30 \%$ showed a high diagnostic accuracy in predicting AF termination on ablation and freedom from AF/AT during follow-up (CS CLV OR 25.6, AUC 0.91; CS APS OR 15.9, AUC 0.94; proportion of LVZs OR 21.4, AUC 0.88). These markers were independent predictors of AF termination on ablation and $\mathrm{AF} / \mathrm{AT}$ recurrence during follow-up. Ablation of a smaller number of drivers that demonstrate greater dominance strongly correlate with greater CS organization.

Conclusions- Novel CS electrogram characteristics were independent predictors of AF termination and $\mathrm{AF} / \mathrm{AT}$ recurrence during follow-up. These markers can potentially aid in predicting outcomes and guide ablation and follow-up strategies.

Key words- Atrial fibrillation, Coronary sinus, Catheter ablation, Mapping, Drivers

\section{INTRODUCTION}

The success rate of catheter ablation for persistent atrial fibrillation (AF) remains suboptimal ${ }^{1}$. Markers including left atrial (LA) size and AF duration has been utilized in predicting procedural success rate ${ }^{2-5}$. Achieving procedural AF termination has been linked to smaller LA size ${ }^{3}$. Ablation of localized drivers has been utilized as a strategy for persistent $\mathrm{AF}$ ablation ${ }^{6-10}$. Localized drivers with certain electrogram characteristics such as lowest cycle length variability (CLV) and highest regularity index (RI) has shown to be associated with AF termination on ablation ${ }^{11}$. The role of coronary sinus (CS) electrogram characteristics in predicting $\mathrm{AF}$ termination and freedom from $\mathrm{AF}$ /atrial tachycardia (AT) during follow-up has not yet been evaluated.

Stochastic Trajectory Analysis of Ranked Signals (STAR) is a novel-mapping method for identifying localized drivers that potentially play a mechanistic role in maintaining AF. The STAR mapping method uses data on multiple individual wavefront trajectories to identify atrial regions that most often precede activation of neighboring areas with the aim of identifying intermittent drivers ${ }^{7,12}$. Pulmonary vein isolation (PVI) plus STAR mapping guided ablation using both global mapping with whole chamber basket catheters and 
sequential mapping with multipolar catheter has been associated with high freedom from AF/AT during long-term follow-up 7,1314 .

The aim of the study was to include patients that underwent ablation guided by STAR mapping in addition to PVI and determine whether CS electrogram characteristics play a role in predicting AF termination on ablation and freedom from AF/AT during follow-up.

\section{METHODS}

\section{STAR mapping principals}

The STAR mapping method has been described in detail in previously published work ${ }^{6,7,12}$. In brief, the principle of STAR mapping is to use data from multiple individual wavefront trajectories to identify regions of the atrium that most often precede activation of neighboring areas. By gathering data from many hundreds of activations, a statistical model can be formed. This permits regions of the atrium to be ranked according to the amount of time that activations precede those of adjacent regions (Supplemental Methods).

\section{STAR mapping cohort}

Patient selection

Patients with persistent AF were included (AF duration $<24$ months and no previous AF ablation). All patients provided informed consent for their study participation. Ethical approval was granted by the UK National Research Ethics System (16/LO/1379). The study was prospectively registered on clinicaltrials.gov (NCT02950844).

\section{STAR mapping ablation}

The ablation protocol and STAR mapping unipolar recordings has been detailed in previous publications 6,7,15. In brief, all patients underwent PVI with wide area circumferential ablation (WACA) and if AF remained post PVI, STAR maps were created using unipolar recordings obtained either with a whole-chamber basket catheter or sequentially with multipolar catheters. AF drivers (AFD) were targeted based on the STAR maps. A study-defined ablation response was either AF termination or cycle length (CL) slowing of [?]30ms. If AF terminated before other AFD had been ablated, these sites were not empirically targeted. Beyond targeting AFD, no other empirical ablation was allowed including the creation of lines. If AF organized into an AT this was mapped and ablated during the procedure. DC cardioversion was performed at the end of the procedure if AF did not terminate following ablation of all identified AFD (Supplemental Methods).

\section{Predictors of AF termination on ablation}

\section{CS electrogram characteristics}

Unipolar electrogram recordings over 5 minutes from a deca catheter positioned in the CS were utilized for the analysis. The unipolar electrograms were obtained by referencing to the ablation catheter positioned in the IVC. The electrogram characteristics evaluated were CS CLV and CS activation pattern stability (APS). These markers were used to allow assessment of the level of CS organization with regard to both CL and activation pattern. The method used to establish the CS CLV and CS APS is detailed in the Supplemental Methods, Supplemental Figure 2 and Supplemental Figure 3.

The relationship between both CS CLV and CS APS with AF termination as the response to ablation was assessed. The relationship between AFD characteristics and CS electrogram characteristics were also assessed. The localized driver characteristics evaluated included temporal stability and recurrence rate. Temporal stability was defined as the number of consecutive atrial activations during which the AFD was leading surrounding electrodes. Recurrence rate was defined as the proportion of time the AFD was leading surrounding electrodes.

Bipolar voltage 
All patients underwent a detailed bipolar voltage map created with the multipolar mapping catheter. The voltages obtained in the PVs were excluded. The colour fill was set at $5 \mathrm{~mm}$ and low voltage zones (LVZs) were defined as areas with a voltage of $<0.2 \mathrm{mV}$. The relationship between the proportion of the left atrium (LA) that was LVZs and achieving AF termination with ablation was determined.

Other potential predictive markers

LA size was determined in all patients and defined as the LA diameter obtained from the parasternal view on a transthoracic echocardiogram. AF duration was defined as the duration of continuous AF in months. Any underlying structural heart disease was also noted. These additional parameters were included in a multivariate analysis to determine which makers were potential predictive markers of AF termination on ablation.

\section{Predictors of freedom from $A F / A T$}

All patients in the STAR guided ablation cohort underwent clinical follow-up at 3, 6 and 12 months, with 48hour ambulatory Holter monitoring at 6 and 12 months. Patients were followed up 6 monthly thereafter. A 3month "blanking period" was observed, with all medication including anti-arrhythmic drugs continued during this time. Clinical success was defined as freedom from AF / AT lasting $>30$ seconds off anti-arrhythmic drugs subsequent to the 3 month blanking period after a single procedure, as per consensus recommendations ${ }^{16}$. The role of CS electrogram characteristics in predicting freedom from AF/AT was evaluated. The association with the proportion of the LA that was LVZs with freedom from AF/AT was also evaluated.

\section{Statistical analysis}

Statistical analyses were performed using SPSS (IBM SPSS Statistics, Version 24 IBM Corp, NY, USA) and are detailed in Supplemental Methods.

\section{RESULTS}

Baseline characteristics

Sixty-five patients were included in the STAR mapping study (Supplemental Figure 1). Baseline characteristics and general procedural and follow-up data is summarised in Supplemental Table 1. One patient experienced cardiac tamponade that was noted at the end of the procedure that required pericardiocentesis.

\section{Predictors of AF termination on ablation}

Figure 1 demonstrates AF termination on a per patient and driver basis. The average RF time post PVI to acheve AF termination was 6.5+-2.1 minutes. The AFD demonstrated an average proportion of time leading of $86.6+-14.3 \%$. The average number of consecutive atrial activations of which it was leading was $5.3+-0.4$ per driver occurrence. AFD where AF termination occurred with ablation showed a greater temporal stability (i.e. higher number of consecutive atrial activations when leading surrounding electrodes) compared to drivers where CL slowing of [?] $30 \mathrm{~ms}$ or no pre-defined ablation response occurred on ablation (5.8+-0.5 vs. $4.3+-0.6 ; \mathrm{p}=0.002)$. They also showed a higher proportion of time leading surrounding electrodes compared to drivers where CL slowing of [?]30ms occurred on ablation $(79.5+-10.4 \%$ vs. $94.5+-15.6 \%$; $\mathrm{p}=0.006)$.

There was a significant correlation between CS CLV and CS APS ( $\mathrm{R}$ of $-0.84 ; \mathrm{p}<0.001$ ). Therefore in the presence of greater CS activaiton pattern stability the CS CLV was lower. Patients with a lower proportion of LVZs were more likely to have a lower CS CLV $(\mathrm{R}=0.77, \mathrm{p}<0.001)$. Patients with a lower proportion of LVZs were also more likely to have a higher proportion of CS APS $(\mathrm{R}=-0.73, \mathrm{p}<0.001)$. When assessing the relationship between AF duration and CS electrogram characteristics there was no strong correlation between AF duration and CS CLV $(\mathrm{R}=0.24, \mathrm{p}=0.06)$ or $\operatorname{CS}$ APS $(\mathrm{R}=0.24, \mathrm{p}=0.06)$. AF duration also did not show a correlation with proportion of LVZs whereby longer duration of AF did not significantly correlate with higher proportion of LVZs $(\mathrm{R}=-0.19, \mathrm{p}=0.13)$.

CS electrogram characteristics 
Patients with and without AF termination on ablation had a significantly different CS CLV (26.1+-4.6 vs. $41.9+-10.2 ; \mathrm{p}<0.001$ ) (Table 1 ). CS CLV was highly predictive of AF termination on ablation with an AUC of 0.85 (95\%CI $0.70-1.01 ; \mathrm{p}<0.001)$. The optimal cutoff for CS CLV was $<30$ ms which had a sensitivity and specificity of $83.0 \%$ (95\% CI 70.2-91.9) and $83.3 \%$ (95\% CI 51.5-97.9) in predicting AF termination on ablation respectively (Table 2). The odds ratio of AF termination on ablation in a patient with a CS CLV of $<30 \mathrm{~ms}$ was 24.4 (95\% CI 4.6-131.0; $\mathrm{p}<0.001)$.

CS APS was also shown to be significantly different between patients in whom ablation resulted in AF termination versus those it did not $(44.4+-6.6$ vs. $26.5+-8.0 ; \mathrm{p}<0.001)$ (Table 1$)$. CS APS was highly predictive of $\mathrm{AF}$ termination with ablation with an $\mathrm{AUC}$ of $0.86(95 \% \mathrm{CI} 0.82-0.94 ; \mathrm{p}<0.001)$. The optimal cutoff for CS APS was [?]30\% which showed a high sensitivity and specificity in predicting AF termination on ablation (Table 2). The odds ratio of AF termination on ablation with a CS APS of [?]30\% was 26.1 $(95 \%$ CI 5.2-41.4; $\mathrm{p}<0.001)$.

Due to the correlation between CS CLV, CS APS and proportion of LVZs the binary logistic regression analysis was performed seperately with each of these markers. This was to avoid multicollinearity biasing the results. CS CLV and CS APS were both shown to be independent predictors of AF termination on ablation (Table 3, Figure 1A-D and Figure 2A-D).

AFD characteristics were also shown to correlate to CS electrogram characteritics. The average temporal stability of the drivers targeted with ablation in each patient correlated with CS CLV ( $\mathrm{R}=-0.74$. $\mathrm{p}=0.003)$ and CS APS $(\mathrm{R}=0.79, \mathrm{p}<0.001)$. The average recurrence rate of the drivers targeted with ablation in each patient was also shown to correlate with $\operatorname{CS} \operatorname{CLV}(\mathrm{R}=-0.72, \mathrm{p}=0.002)$ and $\operatorname{CS} \operatorname{APS}(\mathrm{R}=0.75, \mathrm{p}<0.001)$. There was also a correlation between the number of AFD ablated and the CS CLV $(\mathrm{R}=0.75, \mathrm{p}<0.001)$ and CS APS $(\mathrm{R}=-0.73, \mathrm{p}<0.001)$ whereby in patients where a lower number of AFD ablated the CS CLV was lower and and the CS APS was higher.

Patients with a CS CLV of $<30 \mathrm{~ms}$ were more likely to have drivers with higher temporal stability (5.9+-0.4 vs. $4.1+-0.5 ; \mathrm{p}<0.001)$ and recurrence rate $(80.5+-9.2 \%$ vs. $95.6+-13.4 \% ; \mathrm{p}<0.001)$ than those patients with a CS CLV of $>30 \mathrm{~ms}$. These findings also applied to CS APS whereby patients with a CS APS of $>30 \mathrm{~ms}$ were more likely to have drivers with higher temporal stability $(6.0+-0.5$ vs. $4.0+-0.7 ; \mathrm{p}<0.001)$ and recurrence rate $(81.5+-11.2 \%$ vs. $96.2+-16.1 \%$; $=0.006)$ than those patients with a CS APS of $<30 \mathrm{~ms}$.

The number of drivers ablated was also significantly lower in patients with a CS CLV of $<30 \mathrm{~ms}$ compared to those with a CS CLV or $>30 \mathrm{~ms}(2.1+-0.6$ vs. $3.0+-0.8 ; \mathrm{p}=0.01)$. This was also true for a CS APS of $>30 \mathrm{~ms}(2.3+-0.5$ vs. $3.1+-0.8 ; \mathrm{p}=0.01)$.

Proportion of LVZs

There was a significant difference in the proportion of LVZs in those patients in whom AF termination was achieved on ablation and those patients it did not $(22.9+-5.6 \%$ vs. $43.5+-9.9 \% ; \mathrm{p}<0.001)$ (Table 1$)$. This was highly predictive of AF termination on ablation with an AUC of 0.85 (95\% Ci 0.81-0.92; $<<0.001)$. The optimal cutoff was $<30 \%$ which showed a high sensitivity and specificity (Table 2). A proportion of LVZs of $<30 \%$ showed an odds ratio of 37.7 (95\% CI 6.9-52.5; $\mathrm{p}<0.001)$ for predicting termination of AF with ablation. On multivariate analysis the proportion of LVZs was an independent predictor of AF termination on ablation (Table 3).

\section{Other markers}

The other makers assessed as per Table 1 including AF duration and LA size were not significantly different between patients in whom AF termination was achieved on ablation versus those it did not. Also these makers showed no significant odds ratios in predicting AF termination on ablation (Supplemental Table 2). AF duration of $<12$ months, LA size $<4 \mathrm{~cm}$ and being on anti-arrhythmic drugs had a low sensitivity and specificity in predicting AF termination on ablation (Table 2). They were also not independent predictors of AF termination (Table 3). There was no difference in the AF termination rates between patients with an $\mathrm{AF}$ duration $<12$ months compared to those with and AF duration between 12-24 months $(\mathrm{p}=0.56)$. 


\section{Predictors of freedom from $A F / A T$ during follow-up}

The average follow-up was 29.5+-3.7 months. Out of the 65 patients, $52(80 \%)$ were free from AF/AT during follow-up. Of the 13 patients in whom AF/AT reoccurred during follow-up, 4 had recurrence of AF and 9 had recurrence of AT. Patients in whom AF termination was achieved with ablation were more likely to be free from $\mathrm{AF} / \mathrm{AT}$ during follow-up compared to those patients in whom AF termination was not achieved with ablation $(48 / 51$ vs. $4 / 14 ; \mathrm{p}<0.001)$. AF termination showed an odds ratio of 40.0 (95\% CI 7.7-207.2; $\mathrm{p}<0.001)$ for predicting freedom from AF/AT during follow-up. On Kaplan-Meier analysis, the survival free from $\mathrm{AF} / \mathrm{AT}$ was significantly higher in patients in whom $\mathrm{AF}$ termination was achieved with ablation compared to those in whom AF termination was not achieved with ablation (log rank test $\mathrm{p}<0.001$ ).

\section{CS electrogram characteristics}

Patients with and without AF/AT recurrence during follow-up had a significantly different CS CLV (41.9+7.7 vs. $25.4+-4.0 ; \mathrm{p}<0.001$ ) (Table 4 ) with CS CLV being lower in patients without $\mathrm{AF} / \mathrm{AT}$ recurrence during follow-up. CS CLV showed a high diagnostic accuracy in predicting freedom from AF/AT during follow-up with an AUC of 0.91 (95\%CI 0.82-1.00; $<<0.001$ ). The optimal cutoff for CS CLV was $<30 \mathrm{~ms}$ which had a sensitivity and specificity of $88.5 \%$ (95\%CI 76.6-95.7) and 76.9\% (95\%CI 46.2-95.0) in predicting $\mathrm{AF}$ termination on ablation respectively. The positive predictive and negative predictive value was $93.9 \%$ (95\% CI 85.0-97.7) and $66.7 \%$ (95\%CI 48.1-81.2) respectively. The odds ratio of freedom from $\mathrm{AF} / \mathrm{AT}$ during follow-up with a CS CLV of $<30 \mathrm{~ms}$ was 25.6 (95\% CI 5.4-119.9; $\mathrm{p}<0.001$ ).

CS APS was also shown to be significantly different between patients with and without AF/AT recurrence during follow-up (26.1+-7.2\% vs. 45.6+-5.5\%; $<<0.001)$ (Table 4). CS APS showed a high degree of accuracy in predicting freedom from $\mathrm{AF} / \mathrm{AT}$ during follow-up with an AUC of $0.94(95 \% \mathrm{CI} 0.88-1.00 ; \mathrm{p}<0.001)$. The optimal cutoff for CS APS was [?]30\% which had a sensitivity and specificity of 93.5\% (95\%CI 82.1-98.6) and $52.6 \%$ (95\%CI 28.9-75.6) respectively. The positive predictive and negative predictive value was $82.7 \%$ (95\%CI 74.7-88.5) and 76.9\% (95\%CI 50.7-91.5) respectively. The odds ratio of freedom from AF/AT during follow-up with a CS APS of [?]30ms was 15.9 (95\%CI 3.6-69.7; $\mathrm{p}<0.001)$.

Due to the correlation between CS CLV, CS APS and proportion of LVZs, Cox regression hazard ratio was again performed seperately with each of these markers. This was to avoid multicollinearity biasing the results. CS CLV and CS APS were shown to be independent predictors of AF/AT recurrence during follow-up (Table 5).

Survival free from AF/AT was significantly higher in patients with a CS APS of [?] $30 \%$ and CS CLV $<30 \mathrm{~ms}$ (Figure 3A-C).

\section{Proportion of LVZs}

There was a significant difference in the proportion of LVZs in those patients with AF recurrence and those without $(42.6+-10.8$ vs. $22.1+-5.8 ; \mathrm{p}<0.001)$ (Table 4$)$. This showed a high diagnostic accuracy in predicting freedom from AF/AT during follow-up with an AUC of 0.88 (95\% CI 0.76-1.00; $\mathrm{p}<0.001)$. The optimal cutoff was $<30 \%$ which showed a sensitivity and specificity of $93.8 \%(95 \% \mathrm{CI} 82.8-98.7)$ and $58.8 \%$ (95\% CI 32.9 81.6) respectively. Positive and negative predictive value was $86.5 \%(95 \% \mathrm{CI} 78.4-91.9)$ and $76.9 \%(95 \% \mathrm{CI}$ 51.0-91.5). A proportion of LVZs of $<30 \%$ showed an odds ratio of $21.4(95 \% \mathrm{CI} 4.7-97.6$; $\mathrm{p}<0.001)$ in predicting freedom from AF/AT during follow-up. Proportion of LVZs was shown to be an independent predictor of $\mathrm{AF} / \mathrm{AT}$ recurrence during follow-up (Table 5). Survival free from AF/AT was significantly higher in patients with a proportion of $\mathrm{LVZ}<30 \%$ (Figure 3A-C).

\section{Other markers}

The other makers assessed as per Table 4 including AF duration and LA size were not significantly different between patients in whom there was $\mathrm{AF} / \mathrm{AT}$ recurrence during follow-up compared to those that did not have $\mathrm{AF} / \mathrm{AT}$ recurrence during follow-up. None of these markers were shown to be independent predictors of AF/AT recurrence during follow-up (Table 5). There was no difference in the AF/AT recurrence rate 
between patients with an AF duration between $<12$ months and those with and AF duration between 12-24 months $(\mathrm{p}=0.67)$.

\section{DISCUSSION}

This study demonstrates that novel CS electrogram characteristics including CS CLV and CS APS, in addition to the proportion of LVZs are independent predictors of achieving AF termination on ablation and freedom from AF/AT during long-term follow-up with a high degree of accuracy. These markers are far more effective in identifying those patients that are more likely to have successful outcomes from a persistent AF catheter ablation than markers such as LA size and AF duration that are currently widely used to predict procedural outcomes and may influence ablation strategies utilized. The study has also shown that the characteristics of CS electrogram correlate strongly with the proportion of LA LVZs, the number of localized drivers identified, and the temporal stability and recurrence rate of identified drivers.

\section{CS electrogram organization and outcome.}

Previous studies have shown that drivers with lower CS CLV or higher RI are more likely to be associated with $\mathrm{AF}$ termination on ablation ${ }^{11,17}$ highlighting the importance of electrogram organization in regards to mechanisms maintaining AF. This is further supported by the findings of this study whereby novel markers measuring the level of organization in the CS was shown to be independent predictors of AF termination with ablation and clinical success at long term follow-up. This is the first study that has evaluated CS characteristics as a predictor of procedural outcomes. In this study, a CS CLV of $<30 \mathrm{~ms}$ and CS activation pattern stability of [?] $30 \%$ were associated with a high odds ratio in predicting AF termination on ablation and freedom from AF/AT during follow-up. Both of these markers were highly predictive of AF termination with a significant AUC.

\section{CS electrogram organization and driver properties.}

Further to this, greater CS organization was also associated with a smaller number of AFD identified, greater diver temporal stability and higher recurrence rate, which have previously been shown to be driver characteristics that are associated with AF termination on ablation ${ }^{11}$. This finding is physiologically plausible in that a smaller number of drivers demonstrating greater dominance in terms of higher temporal stability and recurrence rate are more likely to activate the CS in a consistent manner than a larger number of less dominant drivers. This would explain why AF driver properties correspond to CS electrogram characteristics, why each have implications for AF mechanisms, and why both are linked to AF termination on ablation and clinical outcome.

\section{AF termination as an end-point.}

In this study, it was shown that AF termination on ablation strongly correlated with freedom from $\mathrm{AF} / \mathrm{AT}$ during follow-up. The role of AF termination on ablation in predicting procedural outcomes has shown conflicting evidence ${ }^{2,18-21}$. However, studies that have assessed the role of AF termination as a predictor of freedom from $\mathrm{AF}$ / AT have all been limited to a stepwise ablation strategy with CFAE and/or linear ablation

22. Further to this, it has been argued that the conflicting evidence regarding AF termination on ablation as predictor of outcomes relies on the timing at which AF termination is achieved.

Studies in which AF termination has been an independent predictor of outcomes has arguably been where a majority of patients terminated early on during catheter ablation ${ }^{1,18,21}$ and shorter ablation duration has shown to be an independent predictor of clinical success ${ }^{3}$. In this study, the average duration of ablation post PVI was around 7 minutes with a total ablation duration during AF of around 60 minutes. This is compatible with the duration labeled as termination "early in the ablation procedure" making this study finding consistent with these previous studies that have shown that AF termination is a predictor of clinical outcome if it occurs early on during the ablation. These findings further emphasizes that ablation strategies incorporating driver mapping aiming to terminate $\mathrm{AF}$ with limited ablation are perhaps most likely to obtain the best clinical outcome. 
Relationship between LVZ, CS organization, and other clinical factors.

In this study, it was shown that there was no significant correlation between proportion of LVZs and AF duration, which is supported by the findings of previous studies ${ }^{23}$. Similarly, the there was no correlation between LVZs and LA size or other risk factors for AF progression. CS electrogram characteristics also did not correlate with AF duration, LA size or other risk factors for AF progression, whilst they did correlate significantly with proportion of LA LVZs. These findings highlight several important points. Firstly, AF duration, LA size and other risk factors for AF progression does not predict the degree of LA structural and electrical remodeling in regards to bipolar voltage and the level of CS organization. Secondly, the level of LA remodeling based on bipolar voltage has a direct impact on the electrical characteristics in the LA. This has been supported by previous studies, which have shown that baseline CV, and CV dynamics in the LA differs depending on the underlying bipolar voltage ${ }^{24,25}$. Further to this, proportion of LVZs were shown in this study to be a strong independent predictor of $\mathrm{AF}$ termination and $\mathrm{AF} / \mathrm{AT}$ recurrence during follow-up whereby patients with a proportion of LVZs $<30 \%$ were more likely to have AF termination on ablation and no AF/AT recurrence during follow-up. These findings are supported by that of previous studies, which have shown that presence of LA scarring is a predictor of AF recurrence ${ }^{23}$.

$L V Z s, C S$ organization and current categorization of $A F$.

Currently AF duration is the only parameter used to define AF types into paroxysmal, persistent and longstanding persistent AF. However, in this study AF duration was not shown to be an independent predictor of either AF termination on ablation or AF/AT recurrence during follow-up, which is supported by the findings of previous studies ${ }^{5}$. Studies which have shown that AF duration is a predictor of clinical success have not adjusted for additional markers such as proportion of LVZs and CS electrogram characteristics that were shown to be independent predictors in this study ${ }^{219}$. It is possible that the impact of LA size and AF duration observed in previous studies may have been driven by outliers in terms of patients with very longstanding persistent AF or massively dilated atria who may no longer be selected for ablation. It is important to note that all patients in this study had AF durations of less than 24 months with an average AF duration of around 14 months, and atria were moderately dilated. Smaller differences in LA size and AF duration may be less relevant to outcome. This study indicates that differences in AF duration below 24 months does not independently lead to differences in electrophysiological endpoints and procedural outcomes, although AF durations of greater than 24 months may still be relevant.

Currently AF is defined as early persistent if the AF duration is $<12$ months whilst AF duration of $>12$ months is defined as longstanding persistent $\mathrm{AF}{ }^{16}$. Based on the findings from this study, there was no difference in $\mathrm{AF}$ termination and $\mathrm{AF} / \mathrm{AT}$ recurrence rate between patients with an AF duration $<12$ months and patients with an AF duration of $>12$ months. Therefore, the current categorization of AF in this way is not useful prognostically. AF may be more usefully categorized using measures outlined here which are prognostically useful. Whilst the proportion of LA LVZs was similarly useful, this requires a mapping system, expensive mapping catheters and time to acquire a voltage map. It is feasible that CS electrograms could provide equivalent data as a stand-alone diagnostic test, or at the time of simple ablation using a single shot technology, or perhaps at the start of a case to plan the ablation strategy.

Several studies have proposed that cardiac MRI has the ability to evaluate atrial scar and remodeling and show a strong correlation to the bipolar voltage maps obtained during a catheter ablation ${ }^{26-28}$. Again, it is possible that this data could be derived quickly and cheaply from a CS electrogram.

\section{Limitations}

This study has several important limitations. Firstly, the STAR mapping cohort consists of a relatively small number of patients. However, this cohort of 65 patients was thought reasonable as a mechanistic study using a novel mapping technique. The number of patients undergoing persistent AF ablation is similar to previously reported studies using a novel mapping system ${ }^{29,30}$. Furthermore, all patients underwent driver ablation guided by STAR mapping and it is unclear whether these results apply to other contexts such as conventional ablation or PVI alone for AF, or simply cardioversion. 


\section{CONCLUSIONS}

Novel CS electrogram characteristics including CS CLV and CS APS and proportion of LVZs were shown to be strong independent predictors of AF termination on ablation and $\mathrm{AF} / \mathrm{AT}$ recurrence during follow-up. These three makers were far superior to AF duration and LA size in predicting outcome. Driver characteristics were also closely intertwined with CS electrogram characteristics whereby a smaller proportion of drivers with greater dominance were associated with greater CS organization. CS electrogram organization may be a surrogate for LVZs and therefore presumably CMR assessment of scar, and may be useful in predicting outcome and planning ablation strategy. AF categorization based on organization may be clinically more useful than current definitions with regards to outcomes.

\section{FUNDING}

British Heart Foundation project grant (PG/16/10/32016).

\section{DISCLOSURES}

Prof. Schilling has received speaker and travel grants from Biosense Webster and research grants from Biosense Webster and Boston Scientific. Prof. Hunter has received speaker fees, educational and research grants from Medtronic and Biosense Webster. Dr Honarbakhsh, Prof Schilling, Dr Finlay, and Prof. Hunter are shareholders in Rhythm AI Ltd.

\section{REFERENCES}

1. Verma A, Jiang CY, Betts TR, et al. Approaches to catheter ablation for persistent atrial fibrillation. The New England journal of medicine. 2015;372(19):1812-1822.

2. Scherr D, Khairy P, Miyazaki S, et al. Five-year outcome of catheter ablation of persistent atrial fibrillation using termination of atrial fibrillation as a procedural endpoint. Circulation Arrhythmia and electrophysiology. 2015;8(1):18-24.

3. Heist EK, Chalhoub F, Barrett C, Danik S, Ruskin JN, Mansour M. Predictors of atrial fibrillation termination and clinical success of catheter ablation of persistent atrial fibrillation. The American journal of cardiology. 2012;110(4):545-551.

4. Dittrich HC, Erickson JS, Schneiderman T, Blacky AR, Savides T, Nicod PH. Echocardiographic and clinical predictors for outcome of elective cardioversion of atrial fibrillation. The American journal of cardiology. 1989;63(3):193-197.

5. McCready JW, Smedley T, Lambiase PD, et al. Predictors of recurrence following radiofrequency ablation for persistent atrial fibrillation.Europace: European pacing, arrhythmias, and cardiac electrophysiology: journal of the working groups on cardiac pacing, arrhythmias, and cardiac cellular electrophysiology of the European Society of Cardiology. 2011;13(3):355-361.

6. Honarbakhsh S, Schilling RJ, Finlay M, Keating E, Hunter RJ. Prospective STAR-Guided Ablation in Persistent Atrial Fibrillation Using Sequential Mapping With Multipolar Catheters. Circulation Arrhythmia and electrophysiology. 2020;13(10):e008824.

7. Honarbakhsh S, Hunter RJ, Ullah W, Keating E, Finlay M, Schilling RJ. Ablation in Persistent Atrial Fibrillation Using Stochastic Trajectory Analysis of Ranked Signals (STAR) Mapping Method. JACC Clin Electrophysiol. 2019;5(7):817-829.

8. Haissaguerre M, Hocini M, Denis A, et al. Driver domains in persistent atrial fibrillation. Circulation. 2014;130(7):530-538.

9. Honarbakhsh S, Schilling RJ, Dhillon G, et al. A Novel Mapping System for Panoramic Mapping of the Left Atrium: Application to Detect and Characterize Localized Sources Maintaining Atrial Fibrillation. JACC Clin Electrophysiol. 2018;4(1):124-134. 
10. Knecht S, Sohal M, Deisenhofer I, et al. Multicentre evaluation of non-invasive biatrial mapping for persistent atrial fibrillation ablation: the AFACART study. Europace : European pacing, arrhythmias, and cardiac electrophysiology : journal of the working groups on cardiac pacing, arrhythmias, and cardiac cellular electrophysiology of the European Society of Cardiology.2017;19(8):1302-1309.

11. Honarbakhsh S, Schilling RJ, Providencia R, et al. Characterization of drivers maintaining atrial fibrillation: Correlation with markers of rapidity and organization on spectral analysis. Heart rhythm.2018;15(9):1296-1303.

12. Honarbakhsh S, Hunter RJ, Finlay M, et al. Development, in vitro validation and human application of a novel method to identify arrhythmia mechanisms: The stochastic trajectory analysis of ranked signals mapping method. J Cardiovasc Electrophysiol.2019;30(5):691-701.

13. Honarbakhsh S, Schilling RJ, Finlay M, Keating E, Ullah W, Hunter RJ. STAR mapping method to identify driving sites in persistent atrial fibrillation: Application through sequential mapping. J Cardiovasc Electrophysiol. 2019;30(12):2694-2703.

14. Honarbakhsh S, Schilling R, Finlay M, Keating E, Hunter R. Prospective STAR guided ablation in persistent Af using sequential mapping with multipolar catheters Circulation Arrhythmia and electrophysiology. 2020 .

15. Honarbakhsh S, Schilling RJ, Providencia R, et al. Ablation guided by STAR-mapping in addition to pulmonary vein isolation is superior to pulmonary vein isolation alone or in combination with CFAE/linear ablation for persistent AF. J Cardiovasc Electrophysiol.2021;32(2):200-209.

16. Calkins H, Hindricks G, Cappato R, et al. 2017 HRS/EHRA/ECAS/APHRS/SOLAECE expert consensus statement on catheter and surgical ablation of atrial fibrillation. Heart rhythm.2017;14(10):e275-e444.

17. Honarbakhsh S, Schilling RJ, Keating E, Finlay M, Hunter RJ. Drivers in AF colocate to sites of electrogram organization and rapidity: Potential synergy between spectral analysis and STAR mapping approaches in prioritizing drivers for ablation. J Cardiovasc Electrophysiol. 2020;31(6):1340-1349.

18. O'Neill MD, Wright M, Knecht S, et al. Long-term follow-up of persistent atrial fibrillation ablation using termination as a procedural endpoint. European heart journal.2009;30(9):1105-1112.

19. Rostock T, Salukhe TV, Steven D, et al. Long-term single- and multiple-procedure outcome and predictors of success after catheter ablation for persistent atrial fibrillation. Heart rhythm.2011;8(9):13911397.

20. Elayi CS, Di Biase L, Barrett C, et al. Atrial fibrillation termination as a procedural endpoint during ablation in long-standing persistent atrial fibrillation. Heart rhythm.2010;7(9):1216-1223.

21. Kochhauser S, Jiang CY, Betts TR, et al. Impact of acute atrial fibrillation termination and prolongation of atrial fibrillation cycle length on the outcome of ablation of persistent atrial fibrillation: A substudy of the STAR AF II trial. Heart rhythm.2017;14(4):476-483.

22. Lim HS, Derval N, Komatsu Y, et al. Is ablation to termination the best strategy for ablation of persistent atrial fibrillation? Persistent atrial fibrillation is best ablated by a strategy that terminates the arrhythmia: procedural termination is associated with improved long-term outcomes. Circulation Arrhythmia and electrophysiology.2015;8(4):963-971.

23. Verma A, Wazni OM, Marrouche NF, et al. Pre-existent left atrial scarring in patients undergoing pulmonary vein antrum isolation: an independent predictor of procedural failure. Journal of the American College of Cardiology. 2005;45(2):285-292.

24. Honarbakhsh S, Schilling RJ, Orini M, et al. Left atrial scarring and conduction velocity dynamics: Rate dependent conduction slowing predicts sites of localized reentrant atrial tachycardias. International journal of cardiology. 2019;278:114-119. 
25. Honarbakhsh S, Schilling RJ, Orini M, et al. Structural remodeling and conduction velocity dynamics in the human left atrium: Relationship with reentrant mechanisms sustaining atrial fibrillation. Heart rhythm. $2019 ; 16(1): 18-25$.

26. Kalman JM, Kistler PM, Taylor AJ. Cardiac magnetic resonance imaging to detect non-contiguous scar following atrial fibrillation ablation: identifying our knowledge gaps. European heart journal.2014;35(22):1436-1438.

27. Caixal G, Alarcon F, Althoff TF, et al. Accuracy of left atrial fibrosis detection with cardiac magnetic resonance: correlation of late gadolinium enhancement with endocardial voltage and conduction velocity.Europace : European pacing, arrhythmias, and cardiac electrophysiology : journal of the working groups on cardiac pacing, arrhythmias, and cardiac cellular electrophysiology of the European Society of Cardiology. 2020 .

28. Zghaib T, Keramati A, Chrispin J, et al. Multimodal Examination of Atrial Fibrillation Substrate: Correlation of Left Atrial Bipolar Voltage Using Multi-Electrode Fast Automated Mapping, Point-by-Point Mapping, and Magnetic Resonance Image Intensity Ratio. JACC Clin Electrophysiol. 2018;4(1):59-68.

29. Narayan SM, Krummen DE, Shivkumar K, Clopton P, Rappel WJ, Miller JM. Treatment of atrial fibrillation by the ablation of localized sources: CONFIRM (Conventional Ablation for Atrial Fibrillation With or Without Focal Impulse and Rotor Modulation) trial. Journal of the American College of Cardiology. 2012;60(7):628-636.

30. Shivkumar K, Ellenbogen KA, Hummel JD, Miller JM, Steinberg JS. Acute termination of human atrial fibrillation by identification and catheter ablation of localized rotors and sources: first multicenter experience of focal impulse and rotor modulation (FIRM) ablation.J Cardiovasc Electrophysiol. 2012;23(12):1277-1285.

\section{FIGURE LEGEND}

Figure $1 \boldsymbol{A}-\boldsymbol{D}$ - Demonstrates $\boldsymbol{A}$ - STAR map in a posterior-anterior view that shows an AFD on the high posterior wall.B-Ablation here as shown on the CARTO map in an a posterior-anterior view resulted in AF termination to an $A T$ as shown on the $C$ - BARD electrograms including PV signals, CS signals, ablation catheter signals (Map) and surface ECG. D-BARD electrograms demonstrating a proportion of the unipolar CS signals obtained during the 5-minute recording post PVI. The electrograms demonstrate a stable CS activation pattern whereby CS 5 (highlighted by a star) is leading its neighboring electrodes as shown by the orange arrows. Throughout the 5-minute recording, this activation pattern was present for $42 \%$ of the time i.e. the activation pattern stability was $42 \%$. As shown on the CS electrograms there was minimal CL variation within the CS with a CLV or 21 ms during the 5-minute recording.

Figure 2A-D-Demonstrates $\boldsymbol{A}$ - STAR map in an anterior-posterior view that shows an AFD on the mid anterior wall.B-Ablation here as shown on the CARTO map in an anterior-posterior view resulted in AF termination to an $A T$ as shown on the $C$ - BARD electrograms including PV signals, CS signals, ablation catheter signals (Map) and surface ECG. D-BARD electrograms demonstrating a proportion of the unipolar CS signals obtained during the 5-minute recording post PVI. The electrograms demonstrate a stable CS activation pattern whereby CS 10 (highlighted by a star) is leading its neighboring electrodes as shown by the orange arrows. Throughout the 5-minute recording, this activation pattern was present for $45 \%$ of the time i.e. the activation pattern stability was $45 \%$. As shown on the electrograms there was minimal CL variation within the CS with a CLV of 19.9 ms during the 5-minute recording.

Figure 3A-C-Demonstrates Kaplan Meier curves that show a significant difference in survival free from $A F / A T$ in patients based on differences in $\boldsymbol{A}$ - CS activation pattern stability whereby patients with a CS activation pattern stability of [?]30\% have an increase survival free from AF/AT compared to patients with a CS activation pattern stability of $<30 \%$. B-CS CLV whereby patients with a CS CLV of <30ms have an increase survival free from AF/AT compared to patients with a CS CLV of [?]30ms.C-Proportion of LVZs whereby patients with a proportion of $L V Z s$ of $<30 \%$ have an increase survival free from $A F / A T$ compared to patients with a proportion of LVZs of [?]30\%. 
Table 1- Demonstrates the differences in potential predictors between the group with AF termination on ablation versus the group without AF termination on ablation.

\begin{tabular}{|c|c|c|c|c|c|}
\hline \multicolumn{2}{|l|}{ Potential predictors } & \multicolumn{2}{|c|}{ AF termination on ablation $\mathrm{n}=51$} & \multicolumn{2}{|c|}{ No AF termination on ablation } \\
\hline \multicolumn{2}{|c|}{$\mathrm{CS} \mathrm{CLV}^{*}$ ms mean $\pm \mathrm{SD}$} & \multicolumn{2}{|l|}{$26.1 \pm 4.6$} & \multicolumn{2}{|c|}{$41.9 \pm 10.2$} \\
\hline \multicolumn{2}{|c|}{$\mathrm{CS}$ activation pattern stability $\%$ mean $\pm \mathrm{SD}$} & \multicolumn{2}{|l|}{$44.4 \pm 6.6$} & \multicolumn{2}{|c|}{$26.5 \pm 8.0$} \\
\hline \multicolumn{2}{|c|}{ Proportion of LVZs $\%$ mean \pm SD } & \multicolumn{2}{|l|}{$22.9 \pm 5.6$} & \multicolumn{2}{|c|}{$43.5 \pm 9.9$} \\
\hline \multicolumn{2}{|c|}{$\mathrm{AF}$ duration months mean $\pm \mathrm{SD}$} & \multicolumn{2}{|l|}{$14.4 \pm 5.2$} & \multicolumn{2}{|c|}{$11.7 \pm 5.3$} \\
\hline \multicolumn{2}{|c|}{$\mathrm{LA}$ size $\mathrm{cm}$ mean $\pm \mathrm{SD}$} & \multicolumn{2}{|l|}{$4.1 \pm 0.4$} & \multicolumn{2}{|c|}{$3.9 \pm 0.4$} \\
\hline \multicolumn{2}{|c|}{ Anti-arrhythmic drugs n (\%) } & \multicolumn{2}{|l|}{$36(70.6)$} & \multicolumn{2}{|c|}{$9(64.3)$} \\
\hline \multicolumn{2}{|c|}{ Age yrs. mean $\pm \mathrm{SD}$} & \multicolumn{2}{|l|}{$61.4 \pm 8.2$} & \multicolumn{2}{|c|}{$59.6 \pm 11.8$} \\
\hline \multicolumn{2}{|c|}{ Male n (\%) } & \multicolumn{2}{|l|}{$39(76.5)$} & \multicolumn{2}{|c|}{$8(57.1)$} \\
\hline \multicolumn{2}{|c|}{ Previous cardiac surgery n (\%) } & \multicolumn{2}{|l|}{$1(2.0)$} & \multicolumn{2}{|c|}{$0(0)$} \\
\hline \multicolumn{2}{|c|}{ Structural heart disease n $(\%)$} & \multicolumn{2}{|l|}{$3(5.9)$} & \multicolumn{2}{|c|}{$1(7.1)$} \\
\hline \multicolumn{2}{|c|}{ CVA n $(\%)$} & \multicolumn{2}{|l|}{$1(2.0)$} & \multicolumn{2}{|c|}{$1(7.1)$} \\
\hline Hypertension n (\%) & & $13(25.5)$ & & $3(21$. & \\
\hline $\begin{array}{l}\text { Table 2- Demonstrate } \\
\text { ablation. }\end{array}$ & the predictive value of & hese parameters & egards to prec & icting & termination on \\
\hline Potential predictors & $\begin{array}{l}\text { Sensitivity \% } \\
(95 \% \mathrm{CI})\end{array}$ & $\begin{array}{l}\text { Specificity \% } \\
(95 \% \mathrm{CI})\end{array}$ & $\begin{array}{l}\text { Positive pres } \\
\text { value } \%(95)\end{array}$ & $\begin{array}{l}\text { lictive } \\
\left.{ }_{0} \mathrm{CI}\right)\end{array}$ & $\begin{array}{l}\text { Negative predictive } \\
\text { value } \%(95 \% \mathrm{CI})\end{array}$ \\
\hline $\mathrm{CS} \mathrm{CLV}^{*}<30 \mathrm{~ms}$ & $83.0(70.2-91.9)$ & $83.3(51.6-97.9)$ & $95.7(86.1-98$ & .7) & $52.6(36.8-68.0)$ \\
\hline $\begin{array}{l}\text { CS activation } \\
\text { pattern stability } \\
>30 \%\end{array}$ & $90.7(79.7-96.9)$ & $72.7(39.0-94.0)$ & $94.2(86.1-97$ & & $61.5(39.2-79.9)$ \\
\hline $\begin{array}{l}\text { Proportion of } \\
\text { LVZs <30\% }\end{array}$ & $85.2(73.9-93.4)$ & $81.8(48.2-97.7)$ & $95.8(86.7-98$ & $8)$ & $52.9(35.9-69.3)$ \\
\hline $\begin{array}{l}\text { AF duration } \\
<12 \text { months }\end{array}$ & $33.3(21.1-47.5)$ & $54.6(23.4-83.3)$ & $83.1(71.7-91$ & $.2)$ & $14.3(8.6-22.8)$ \\
\hline LA size $<4 \mathrm{~cm}$ & $44.4(30.9-58.6)$ & $45.5(16.8-76.6)$ & $83.1(71.7-91$ & $.2)$ & $80.0(68.4-74)$ \\
\hline $\begin{array}{l}\text { Anti-arrhythmic } \\
\text { drugs }\end{array}$ & $72.2(58.4-83.5)$ & $45.5(16.8-76.6)$ & $86.7(78.7-92$ & $.0)$ & $25.0(13.3-42.0)$ \\
\hline
\end{tabular}

Table 3- Binary logistic regression findings. CS CLV, CS activation pattern and proportion of LVZs were incorporated separately with the other assessed parameters and were shown to be independent predictors of AF termination on ablation.

$\begin{array}{ll}\text { Potential Predictors } & \text { Potential Predictors } \\ & C S C L V^{*} \text { Model } \\ & \text { CS CLV } \\ \text { AF duration } \\ \text { LA size } \\ \text { Antiarrhythmic drugs } \\ \text { Age } \\ \text { Male } \\ \text { Previous cardiac surgery } \\ \text { Structural heart disease }\end{array}$

$\begin{array}{ll}\text { Potential Predictors } & \text { P-value } \\ C S C L V^{*} \text { Model } & \\ \text { CS CLV } & <0.001 \\ \text { AF duration } & 0.13 \\ \text { LA size } & 0.24 \\ \text { Antiarrhythmic drugs } & 0.25 \\ \text { Age } & 0.55 \\ \text { Male } & 0.16 \\ \text { Previous cardiac surgery } & 0.25 \\ \text { Structural heart disease } & 0.66\end{array}$




\begin{tabular}{ll} 
AF duration & AF duration \\
LA size & LA size \\
Antiarrhythmic drugs & Antiarrhythmic drugs \\
Age & Age \\
Male & Male \\
Previous cardiac surgery & Previous cardiac surgery \\
Structural heart disease & Structural heart disease \\
CVA & CVA \\
Hypertension & Hypertension \\
Proportion of LVZs Model & Proportion of LVZs Model \\
Proportion of LVZs & Proportion of LVZs \\
AF duration & AF duration \\
LA size & LA size \\
Antiarrhythmic drugs & Antiarrhythmic drugs \\
Age & Age \\
Male & Male \\
Previous cardiac surgery & Previous cardiac surgery \\
Structural heart disease & Structural heart disease \\
CVA & CVA \\
Hypertension & Hypertension \\
\hline
\end{tabular}

CVA

Hypertension

Hypertension

CS activation pattern stability Model

CS activation pattern stability

AF duration

CS activation pattern stability Model

CS activation pattern stability $\quad<0.001$

AF duration $\quad 0.13$

LA size $\quad 0.24$

Antiarrhythmic drugs $\quad 0.25$

Age $\quad 0.55$

Male $\quad 0.16$

Previous cardiac surgery $\quad 0.25$

Structural heart disease $\quad 0.66$

CVA $\quad 0.89$

Hypertension $\quad 0.82$

Proportion of LVZs Model

$<0.001<0.001$

AF duration $\quad 0.13$

LA size $\quad 0.24$

Antiarrhythmic drugs $\quad 0.25$

Age $\quad 0.55$

Male $\quad 0.16$

Previous cardiac surgery $\quad 0.25$

Structural heart disease $\quad 0.66$

CVA $\quad 0.89$

Hypertension 0.82

Table 4-Demonstrates the differences in potential predictors between the group with no AF/AT recurrence during follow-up versus the group with $A F / A T$ recurrence during follow-up.

\begin{tabular}{llll}
\hline Potential predictors & No AF/AT recurrence n=52 & AF $/$ AT recurrence $\mathrm{n}=13$ & P-value \\
\hline CS CLV $^{*}$ ms mean \pm SD & $25.4 \pm 4.0$ & $41.9 \pm 7.7$ & $<0.001$ \\
CS activation pattern stability \% mean \pm SD & $45.6 \pm 5.5$ & $26.1 \pm 7.2$ & $<0.001$ \\
Proportion of LVZs \% mean \pm SD & $22.1 \pm 5.8$ & $42.6 \pm 10.8$ & $<0.001$ \\
AF duration months mean \pm SD & $13.9 \pm 5.4$ & $14.4 \pm 4.8$ & 0.38 \\
LA size cm mean \pm SD & $4.1 \pm 0.4$ & $4.0 \pm 0.4$ & 0.45 \\
Anti-arrhythmic drugs n (\%) & $39(75.0)$ & $7(53.8)$ & 0.18 \\
Age yrs. mean \pm SD & $61.4 \pm 9.0$ & $59.9 \pm 8.6$ & 0.30 \\
Male n (\%) & $38(73.1)$ & $9(69.2)$ & 0.74 \\
Previous cardiac surgery n (\%) & $1(1.9)$ & $0(0)$ & 1.00 \\
Structural heart disease n (\%) & $3(5.8)$ & $1(7.1)$ & 1.00 \\
CVA n (\%) & $2(3.8)$ & $0(0)$ & 1.00 \\
Hypertension n (\%) & $14(26.9)$ & $2(15.4)$ & 0.49 \\
\hline
\end{tabular}

Table 5-Demonstrates the Cox proportional regression analysis in regards to freedom from AF/AT during follow-up. 


AF duration
LA size
Antiarrhythmic drugs
Age
Male
Previous cardiac surgery
Structural heart disease
CVA
Hypertension
Proportion of LVZs Model
Proportion of LVZs
AF duration
LA size
Antiarrhythmic drugs
Age
Male
Previous cardiac surgery
Structural heart disease
CVA
Hypertension

$C S C L V^{*}$ Model
CS CLV
AF duration
LA size
Antiarrhythmic drugs
Age
Male
Previous cardiac surgery
Structural heart disease
CVA
Hypertension
CS activation pattern stability Model
CS activation pattern stability
AF duration
LA size
Antiarrhythmic drugs
Age
Male
Previous cardiac surgery
Structural heart disease
CVA
Hypertension
Proportion of LVZs Model
Proportion of LVZs
AF duration
LA size
Antiarrhythmic drugs
Age
Male
Previous cardiac surgery
Structural heart disease
CVA
Hypertension

CS CLV Model

CS CLV

P-value

AF duration

$<0.001$

LA size

0.72

Antiarrhythmic drugs

0.90

0.94

Age

0.57

Male $\quad 0.85$

Previous cardiac surgery $\quad 0.75$

Structural heart disease $\quad 0.52$

CVA 0.67

Hypertension $\quad 0.44$

CS activation pattern stability Model

CS activation pattern stability $\quad<0.001$

AF duration $\quad 0.72$

LA size $\quad 0.90$

Antiarrhythmic drugs $\quad 0.94$

Age $\quad 0.57$

Male $\quad 0.85$

Previous cardiac surgery $\quad 0.75$

Structural heart disease $\quad 0.52$

CVA 0.67

Hypertension $\quad 0.44$

Proportion of LVZs Model

$<0.001$

$<0.001$

AF duration $\quad 0.72$

LA size $\quad 0.90$

Antiarrhythmic drugs $\quad 0.94$

Age $\quad 0.57$

Male $\quad 0.85$

Previous cardiac surgery $\quad 0.75$

Structural heart disease $\quad 0.52$

CVA 0.67

Hypertension $\quad 0.44$ 

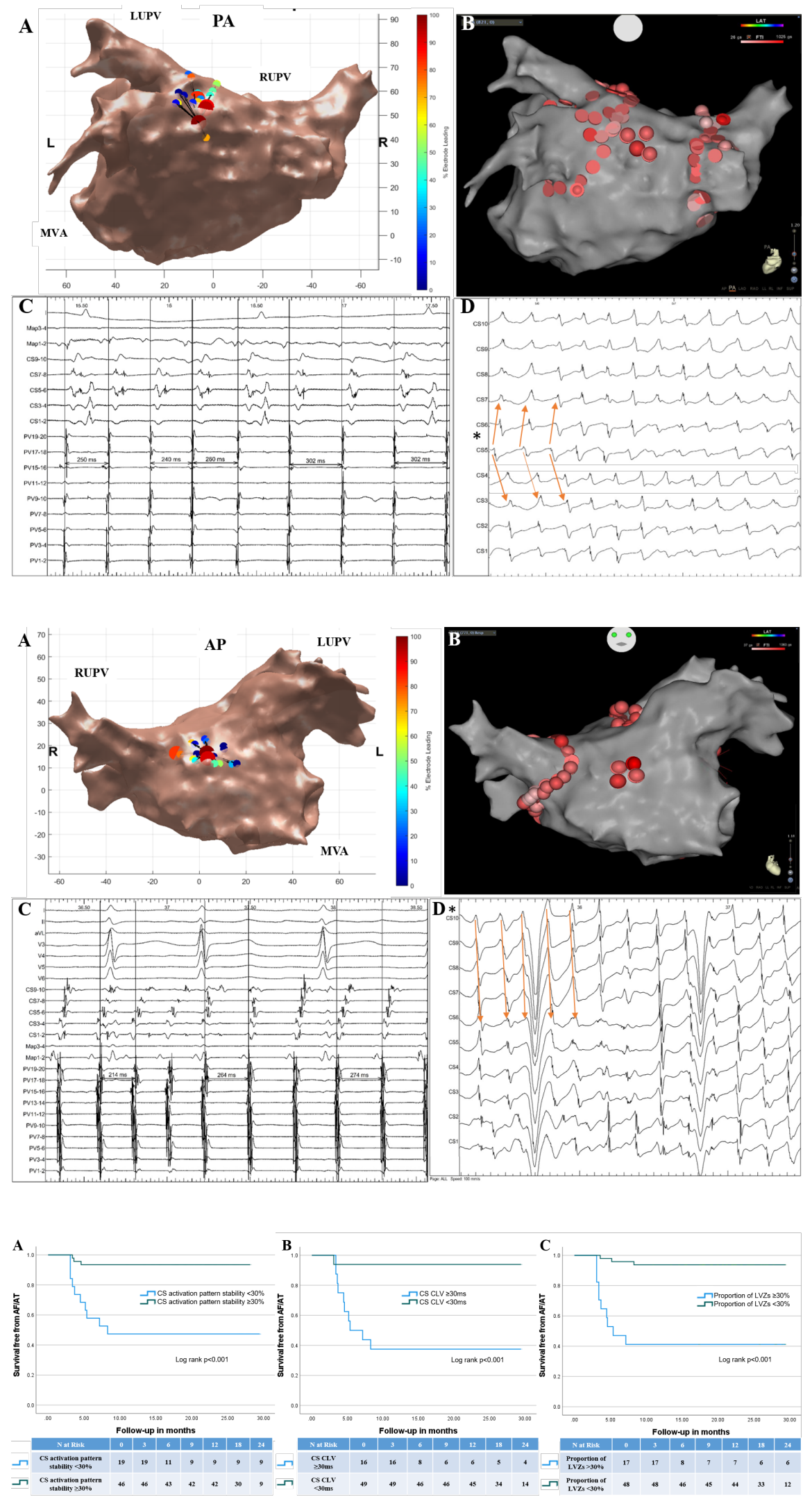